\title{
A Review on Different Techniques to De-noise a Signal
}

\author{
Tanusree Ghosh $^{1}$, Debnath Bhattacharyya ${ }^{1}$, Samir Kumar Bandyopadhyay ${ }^{1}$ \\ and Tai-hoon $\mathrm{Kim}^{2}$ \\ ${ }^{1}$ Department of Computer Science and Engineering, \\ University of Calcutta, Kolkata-700009, India \\ ${ }^{2}$ Department of Convergence Security, Sungshin Women's University, \\ 249-1, Dongseon-dong 3-ga, Seoul, 136-742, Korea \\ \{tanusree1987,debnathb\}@gmail.com,skb1@vsnl.com \\ taihoonn@daum.net (Corresponding Author)
}

\begin{abstract}
This work is based on the comparative study of different decomposition methods used to de-noise an ECG signal. There are several signal processing techniques available like Fourier Transform method, Short Term Fourier Transform method, Wavelet analysis, Empirical Mode of Decomposition method etc. Though Fourier Transform method is predominantly used for decomposition purpose but it is not suitable for decomposition of nonstationary signal. However, most of the biomedical signals are non stationary in nature. So the signal should be decently de-noised thus it can provide essential clinical information relevant to patient's health condition. The difficulties of FT method can be knocked out by Wavelet Transform method. As Wavelet Transform is a non adaptive approach, it is not satisfactory to eliminate the high frequency noise from signal. The complications of WT method can be diminished by Empirical Mode of Decomposition method. A detail study is required to find out the most advantageous decomposition method for an ECG signal.
\end{abstract}

Keywords: ECG, EMD, STFT, signal, DWT

\section{Introduction}

An electrocardiogram (ECG) represents cardiac signals generated by cardiac muscles. It is so far the most common method used by the cardiologist for determining cardiac functions. A typical ECG beat contains wave segments: P (it is the first wave of the electrocardiogram), QRS (it follows $\mathrm{P}$ wave, if the first deflection is negative, it is labeled as $\mathrm{Q}$ wave, first positive deflection is called $R$ wave, negative deflection following $R$ wave is labeled as $S$ wave) and $\mathrm{T}$ (it follows the QRS complex, normal shape of $\mathrm{T}$ wave is slightly rounded and asymmetrical) which represents periodic depolarization and re-polarization of atria and ventricles in a sequential manner.

The timings of the segments or the amplitude of the individual peak provide vital clinical information about the condition of cardiovascular system. For example, a lengthened QT interval (this interval starts at the onset of the QRS and ends at the end of the T wave) is a biomarker of ventricular tachyarrhythmia and a risk factor for sudden death. Any elongation of the PR segment (PR segment begins at the end of the $\mathrm{P}$ wave and ends at the onset of the QRS complex) can indicate one of the heart block conditions, which may require a surgical procedure. Also changes in time durations of other ECG waveforms such as $\mathrm{P}$ wave and QRS complex for their normal values, indicate the problem of cardiovascular system. So the 
analysis of the ECG waveform provides vital information to the cardiologists and helps them to take necessary action about the patients.

The Filtration of ECG signals is very important, so as to, get the parameters of ECG signal (used for recognizing much variability's of heart activity) clear without noise. Cleaned ECG signal gives full detailed information about the electrophysiology of the heart diseases and ischemic changes that may occur. In order to support the clinical decision making, reasoning tool to the (ECG) signal must be clearly represented and filtered, to remove out all noises (Electrode contact noise, Electromyography (EMG) noise etc.) and artifacts (baseline drift, motion artifacts, power line interference, etc.) from the signal. So it needs a hard work for denoising.

Fourier transform is not useful for analysis of a non-stationary signal like ECG [3]. To overcome this deficiency, a modified method Short Term Fourier Transform is used. STFT allows representing the signal in both time and frequency domain through time windowing functions [2]. The window length determines a constant time and frequency resolution. Thus, a shorter time windowing is used in order to capture the transient behavior of a signal, sacrificing the frequency resolution. So wavelet transform is selected to extract the relevant time amplitude information from a signal. Simultaneously, the signal to noise ratio based on prior knowledge of the signal characteristics is also improved. Wavelet transform is capable of providing the time and frequency information simultaneously, hence giving a time frequency representation of the signal [3]. But some basic wavelet filter is used for all data throughout a signal and this will lead to the loss of some important information presented in that signal. This process is non adaptive [7]. Besides Wavelet transform method is poor at processing non linear data. To overcome this problem EMD method is used to analyze multicomponent signal using intrinsic mode functions [4]. The detailed study is described in the latter part.

\section{Previous Works}

\subsection{Fourier Transform}

Fourier analysis breaks down a signal into constituent sinusoids of different frequencies [1]. Another way to think of Fourier analysis is as a mathematical technique for transforming the view of the signal from a time-based one to a frequency-based one which is shown in Figure 1.

$$
\begin{aligned}
& X(f)=\int_{-\alpha}^{\alpha} x(t) * e^{-2 j \pi f t} * d t \\
& x(t)=\int_{-\alpha}^{\alpha} X(f) * e^{2 j \pi f t} * d f
\end{aligned}
$$
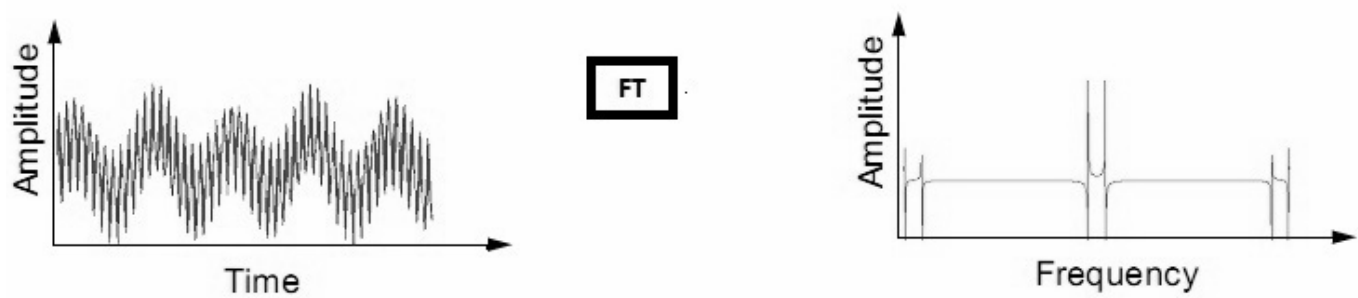

Figure 1. Fourier transform of a time based signal to frequency based signal 
where, $\mathrm{t}$ stands for time, $\mathrm{f}$ stands for frequency, $\mathrm{x}$ denotes the signal in time domain and $\mathrm{X}$ denotes the signal in frequency domain. Equation (1) is called the FT of $x(t)$ and equation (2) is called the inverse FT of $X(f)$.

In the above equation, $t$ stands for time, $f$ stands for frequency, and $x$ denotes the signal at hand. $\mathrm{x}$ denotes the signal in time domain and the $\mathrm{X}$ denotes the signal in frequency domain. This convention is used to distinguish the two representations of the signal. Equation (1) is called the Fourier transform of $x(t)$, and equation (2) is called the inverse Fourier transform of $\mathrm{X}(\mathrm{f})$, which is $\mathrm{x}(\mathrm{t})$. The signal $\mathrm{x}(\mathrm{t})$, is multiplied with an exponential term, at some certain frequency " $\mathrm{f}$ ", and then integrated over all times. The analysis coefficients define the notion of global frequency of a signal. As shown in equation, they are computed as inner products of the signal with sine wave basis functions of infinite duration. As a result, Fourier analysis works well if $\mathrm{x}(\mathrm{t})$ is composed of a few stationary components (e.g., sine waves). However, any abrupt change in time in a non-stationary signal $x(t)$ is spread out over the whole frequency axis. Fourier analysis has a serious drawback in transforming to the frequency domain, time information is lost. When looking at a Fourier transform of a signal, it is impossible to tell when a particular event took place.

\subsection{Short Term Fourier Transform (STFT)}

Short-Time Fourier Transform (STFT), maps a signal into a two-dimensional function of time and frequency. In STFT, a window function with fixed width is chosen [shown in the Figure 2. (a)] and then this window is slid throughout the whole signal [2]. In this case, the signal inside the window is stationary. Then, the inner product of the signal $x(t)$, inside the window is computed. The definition of the STFT is given in one line:

$$
\operatorname{STFT} \stackrel{x}{\omega}^{\omega}(t, f)=\int t^{\prime}\left[x(t) \cdot \omega^{*}\left(t-t^{\prime}\right)\right] * e^{-j 2 \pi f t} d t
$$

$\mathrm{x}(\mathrm{t})$ is the signal, w(t) is the window function, and $*$ is the complex conjugate.

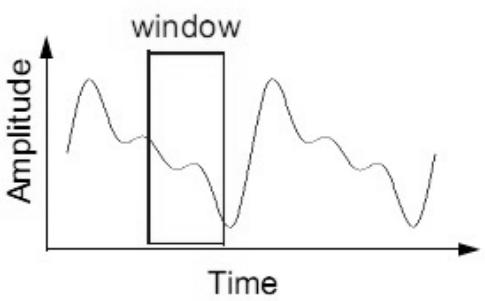

a.

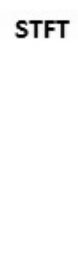

Figure 2 (a). windowing a signal. (b). time frequency resolution in STFT

While the STFT's compromise between time and frequency information can be useful, the drawback is that while choosing a particular size for the time window, that window is the same for all frequencies. It can be said in the following way also [shown in Figure 2(b).]:

Narrow window ===>good time resolution, poor frequency resolution. Wide window $===>$ good frequency resolution, poor time resolution.

The resolution problem of STFT is solved by using wavelet transform which is based on multi-resolution analysis. 


\subsection{Wavelet Transform}

2.3.1. Multi-resolution analysis: Every spectral component is not resolved equally as was the case in the STFT. Multi-resolution analysis [5] is designed to give good time resolution and poor frequency resolution at high frequencies and good frequency resolution and poor time resolution at low frequencies. This concept is meaningful to the signal which has a relatively low frequency component throughout the entire signal and relatively high frequency components for a short duration somewhere around the middle [shown in Figure 3 (b)]. The illustration in Figure 3(a) is used to explain the interpretation of time and frequency resolutions in case of wavelet transform technique.. The certain non-zero area cannot imply the value of a particular point in the time-frequency plane. All the points in the timefrequency plane that falls into a box are represented by one value of the WT.

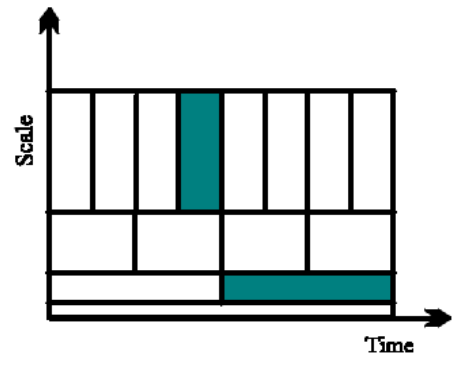

a.Time Frequency resolution of WT

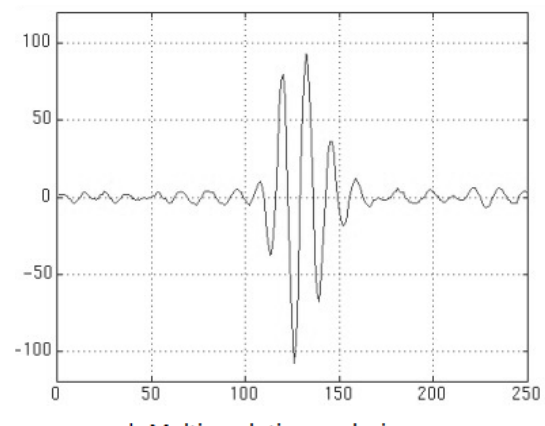

b.Multiresolution analysis

Figure 3 (a). Time-Frequency resolution of wavelet transforms. (b). Multiresolution analysis

2.3.2. The continuous wavelet transform: The CWT can operate at every scale, from that of the original signal up to some maximum scale which can be determined by trading off the need for detailed analysis with available computational horsepower. The CWT is also continuous in terms of shifting: during computation, the analyzing wavelet is shifted smoothly over the full domain of the analyzed function.

2.3.3. Discrete wavelet transform: In continuous wavelet transform coefficient every possible scale is considered and this is a difficult job. So if scales and positions based on powers of two (dyadic scales and positions), can be chosen, then the analysis will be much more efficient and just as accurate. Such an analysis can be obtained from the discrete wavelet transform (DWT). The algorithm is like tree [3] as shown Figure 4. 


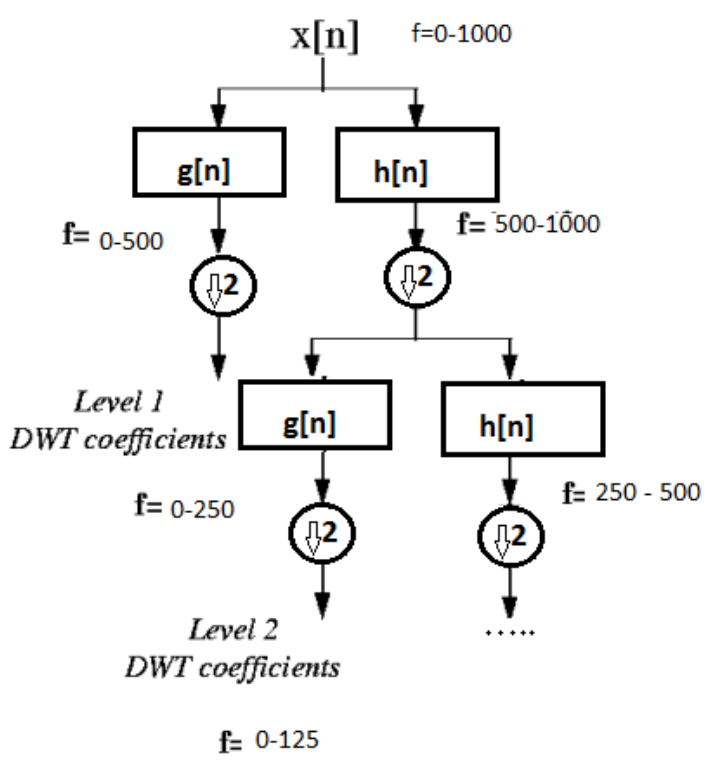

Figure 4. Decomposition using DWT

In the Figure 4, decomposition of a signal (with sampling frequency $1 \mathrm{KHz}$ ) by using dyadic scale DWT is shown. The wavelet decomposition results in levels of approximated and detailed coefficients. The approximation is then itself split into a second-level approximation and detail, and the process is repeated. For n-level decomposition, there are $n+1$ possible ways to decompose or encode the signal. This multi-resolution analysis enables the user to analyze the signal in different frequency bands; therefore, any transient in time domain as well as in frequency domain can be observed.

2.3.4. Wavelet filter: There are different types of wavelet filters and they are used on the basis of the structure of the signal which has to be filtered (e.g., db6 have close similarities with ECG morphology, so db6 used very often to decompose an ECG signal).

As same basic wavelet is used for all data throughout the signal, wavelet transform is a non adaptive approach, which is not suitable to eliminate the high frequency noise from signal. So a complete, orthogonal, local, and adaptive approach is considered, which is called Empirical Mode Decomposition Method.

\subsection{Empirical Mode Decomposition (EMD) Method}

Empirical mode decomposition method is an adaptive time-frequency data analysis method of decomposing a non linear and non stationary natural signal into a definite number of high frequency and low frequency components proposed by Huang et al. The EMD technique is part of a process known as the Hilbert-Huang Transform (HHT) that consists of two main elements: the EMD and the Hilbert spectral analysis. The EMD generates the intrinsic mode functions (IMFs) from the data, and the Hilbert spectral analysis is required to generate a "time-frequency-energy" representation of the data, based on the IMFs [8]. Intrinsic mode functions are used to decompose the time series into superposition of components with well defined instantaneous frequency. 
2.4.1. Instantaneous frequency: Local time/frequency scale can be defined by instantaneous frequency. Instantaneous frequency can be defined as differential coefficient of analytic signal phase function [10]. If we consider $\mathrm{x}(\mathrm{t})$ is a non stationary signal, then the instantaneous frequency of this signal form an analytic signal $\mathrm{Z}(\mathrm{t})$, by taking the Hilbert Transform of $\mathrm{x}(\mathrm{t})$, such as:

$$
\begin{aligned}
Y(t) & =\frac{1}{\pi} * P \int_{-\alpha}^{\alpha} \frac{x\left(t^{\prime}\right)}{t-t^{t}} d t^{t} \ldots \ldots \ldots \ldots \ldots(4) \\
Z(t) & =x(t)+i Y(t)=A(t) * e^{i * \theta(t)} \ldots \ldots(5)
\end{aligned}
$$

where, $\mathrm{Y}(\mathrm{t})$ is the best local fit of a trig function to $\mathrm{x}(\mathrm{t})$ and $\mathrm{Z}(\mathrm{t})$ has the same positive frequency spectrum as the original signal, but zero negative frequency spectrums.

2.4.2. Intrinsic Mode Functions (IMFs): Intrinsic mode function represents the oscillation mode imbedded in the data. The IMF in each cycle, defined by the zero crossings, involves only one mode of oscillation, no complex riding waves are allowed. IMF component follows the basic requirement of completeness, orthogonality, locality, and adaptiveness. An IMF can be obtained successfully by eliminating riding waves and asymmetries locally. IMFs are functions that have the same number of zero crossings and extrema $\&$ the mean value of the upper and the lower envelops is equal to zero [6].

The original signal is decomposed into a sum of intrinsic mode functions (IMFs) by using shifting process. The first separated IMF components have the highest frequency while the finally separated IMF components have the lowest frequency. The remained component is only a monotonic function with only one extreme point. The trend of original signal is represented. The upper and lower envelope curves are partially symmetric with the time axis and any two IMF are independent.

2.4.3. Shifting process: The shifting process [9] is described below:

- All of the local maxima and minima of the original signal are identified first.

- Using cubic spline method, the upper envelope and the lower envelope are produced from local maxima and local minima respectively.

- The mean value $\left(\mathrm{m}_{1}\right)$ of upper envelope and the lower envelope is calculated by averaging these two and this mean value $(\mathrm{m} 1)$ is subtracted from the original signal $(\mathrm{x}(\mathrm{t}))$ to produce the first intrinsic mode function (IMF1) component $\left(\mathrm{h}_{1}(\mathrm{t})\right)$.

$$
\mathrm{h}_{1}(\mathrm{t})=\mathrm{x}(\mathrm{t})-\mathrm{m}_{1}
$$

- If this difference $\left(\mathrm{h}_{1}(\mathrm{t})\right)$ is not an IMF, then the shifting process described in steps (1) and $(2)$, are repeated on the present difference signal $\left(h_{1}(t)\right)$.

$$
h_{11}(t)=h_{1}(t)-m_{11}
$$

$\mathrm{m}_{11}$ is the mean of upper and lower envelope value of $h_{1}$. 
- If after $k$ th. term $\mathrm{h}_{1 \mathrm{k}}$ becomes an IMF, i.e.,

$$
h_{1 k}(t)=h_{1(k-1)}(t)-m_{1 k}
$$

The first residual component $\left(\mathrm{r}_{1}\right)$ is obtained by subtracting the IMF1 component $\left(\mathrm{c}_{1}=\mathrm{h}_{1 \mathrm{k}}\right)$ from the original signal

$$
r_{1}(t)=x(t)-c_{1}(t)
$$

- Then the residual component $t\left(r_{1}\right)$ is treated as a new data and subjected to the same process mentioned above to calculate the next IMF.

$$
\begin{aligned}
\text { i.e., } r_{2}(t)=r_{1}(t)-c_{2}(t) \ldots \ldots(10) & \ldots \ldots \ldots \ldots \\
r_{N}(t)= & r_{(N-1)}(t)-c_{N}(t) \ldots \ldots \ldots(11)
\end{aligned}
$$

- The steps are repeated until the final residual component becomes a monotonic function from which no more IMF can be extracted.

- The EMD of the original signal can be obtained from the equation (9) and (11). And it can be written as

$$
\sum_{\mathrm{n}=1}^{\mathrm{N}} \mathrm{c}_{\mathrm{n}}(\mathrm{t})+\mathrm{r}_{\mathrm{N}}(\mathrm{t})=\mathrm{x}(\mathrm{t}) \ldots \ldots
$$

2.4.4. Reconstruction of IMFs: For the interference suppression of partial discharge signals, the information of partial discharge signals can be clear seen by the way of IMF reconstruction [12]. EMD method starts from the time scale features. It first separates the characteristic time scale in the signals from the smallest modal and then separates the bigger modal of characteristic.

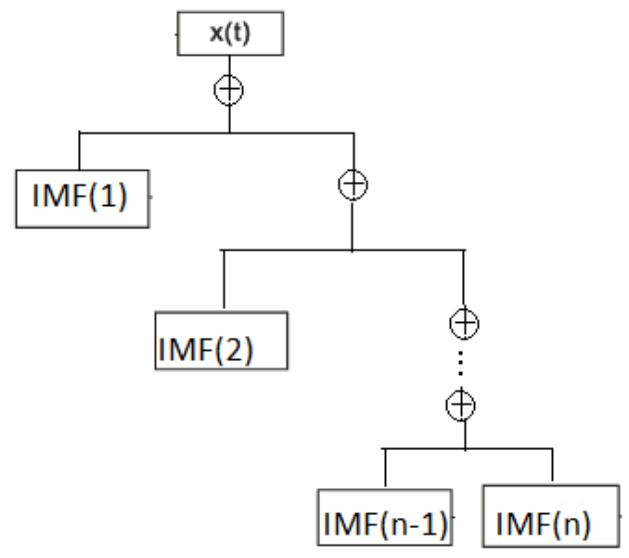

Figure 5. Reconstruction of IMF

Noise signals mainly concentrated in the first few layers of IMF components so signals can be extracted from the details construction from the bottom to the top. Instead of simple partial 
summation of IMFs, different IMFs may be chosen and processed. The tree in the Figure 5 shows the way of reconstruction of the IMF. The analytic expression of $x(t)$ can be obtained after extracting the instantaneous frequency of each IMF component (neglect the $\mathrm{rN}$ term).It can be written as

$$
X(t)=\sum_{j=1}^{n} a_{j}(t) \cdot e^{i \int \omega_{j}(t) d t} \ldots \ldots(13
$$

This is a generalization of the Fourier decomposition, allowing for time varying amplitudes and frequencies, thus simplifying the description of non-stationary data and the spectrum describes the joint distribution of the amplitude and frequency content of the signal as a function of time.

\section{Observation}

In Figure 6, four techniques for filtration of a signal are shown. From the detailed study of these techniques the comparison between these four techniques can be shown in the following way:

A. FT vs. STFT: Fourier transform cannot be used for non-stationary signal (ECG signal is non-stationary) analysis. In case of STFT a window function is selected. A true timefrequency representation can be obtained from the STFT.

B. STFT vs. WT: Every spectral component is not resolved equally in the cases of STFT.A resolution problem is occurred. This problem is solved in WT technique by using the multiresolution approach.

C. WT vs. EMD: In case of using WT, a basic wavelet function (by matching this function with the morphology of the test signal) is selected for all data throughout the test signal. This non-adaptive approach is not suitable to handle the de-noising process for high frequency noises (such as power line interference in ECG signal). This can be improved by using the EMD method.

Compared with Fourier analysis based on Prior function basis and wavelet analysis, EMD does not need to preset primary function. It is a multi-scale time-frequency localization analysis method. The signal in time domain is decomposed by using EMD method into number of IMFs which contain information about the change of frequency of the original signal with time. So, this method overcomes the problem of losing information due to the domain change of the signal. This method is not based on the waveform matching principle. The decomposition effect is not affected by the basic wavelet function selection. This method is complete for a given data set [11]. Local properties of the oscillatory modes are emphasized in this method. It is a complete, orthogonal, local, and adaptive approach.

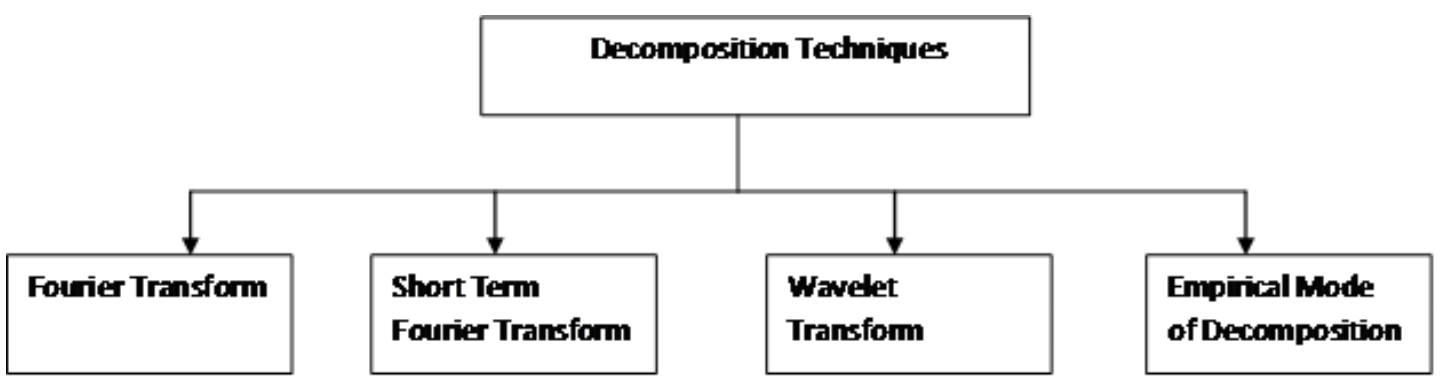

Figure 6. Some techniques used to de-noise a signal 


\section{Conclusion}

In this paper a comparative study between some filtration techniques has been done. As we are mainly interested about ECG signal which is a non-stationary signal, Fourier transform is not suitable for this type of signal. Wavelet Transform technique is very useful to analyze the signal, but as same basic wavelet is used for all data throughout the signal, some high frequency noise cannot be eliminated from the ECG signal by using the same. The nonadaptiveness of this transform method can be overcome by using an adaptive time-frequency data analysis method, called EMD, which also divides frequency according to the physical forms of the signals. From this detail study, it can be concluded that EMD method is superior to the other techniques discussed here to analyze the nonlinear and non-stationary signals.

\section{Future scope}

Real and synthetic noise, baseline wonder in ECG signal can be eliminated from an ECG signal by this EMD method. As EMD technique is an adaptive approach, it may be useful to eliminate the high frequency noise from a nonlinear, non-stationary signal (e.g., ECG signal). A detail study is required to use the EMD method to determine the difference between a fake signal synthesized to look like natural data and a natural signal of the same type.

\section{References}

[1] R. Niu, "Fourier Series and their Applications", (2006) May 12, http://dspace.mit.edu/bitstream/handle/1721.1/78574/18-100c-spring-2006/contents/projects/niu.pdf, (Last accessed on February 19, 2014).

[2] M. Alam, M. I. Islam and M. R. Amin, "Performance Comparison of STFT, WT, LMS and RLS Adaptive Algorithms in Denoising of Speech Signal", IACSIT International Journal of Engineering and Technology, vol. 3, no. 3, (2011) June, pp. 235- 238.

[3] R. Polikar, "The Engineer's ultimate guide to wavelet analysis", The Wavelet Tutorial, IOWA State University of Science and Technology, Part-1 to IV, (1994- 2000), http://users.rowan.edu/ polikar/WAVELETS/WTtutorial.html (Last accessed on February18, 2014).

[4] A. R. Alqawasmi and K. Daqrouq, "ECG Signal Enhancement using Wavelet Transform", WSEAS TRANSACTIONS on BIOLOGY and BIOMEDICINE, Issue 2, vol. 7, (2010) April, pp. 62-71.

[5] M. Kania, M. Fereniec and R .Maniewski, "Wavelet Denoising for Multi-lead High Resolution ECG Signals", MEASUREMENT SCIENCE REVIEW, vol. 7, Section 2, no. 4, (2007), pp. 30- 33, http://www.measurement.sk/2007/S2/Kania.pdf (Last accessed on February01, 2014).

[6] Y. Kopsinis and S. McLaughlin, "Empirical mode decomposition based denoising techniques", 1st IAPR Workshop on Cognitive Information Processing - CIP, Santorini (Thera), Greece, (2008) June 09-10, pp. 4247.

[7] N. ur Rehman and D. P. Mandic, "Filter Bank Property of Multivariate Empirical Mode Decomposition", IEEE TRANSACTIONS ON SIGNAL PROCESSING, vol. 59, no. 5, (2011) May, pp. 2421- 2426.

[8] H. H. Hassan and J. W. Peirce, "Empirical Mode Decomposition (EMD) of potential field data: airborne gravity data as an example", CSEG Recorder, (2008) January, pp. 25- 30.

[9] A. Chacko and S. Ari, "Denoising of ECG signals using Empirical Mode Decomposition based technique", IEEE- International Conference On Advances In Engineering, Science And Management (1CAESM - 2012), (2012) March 30-31, Nagapattinam, Tamil Nadu, India, pp. 6- 9.

[10] J. Jinshui, L. Bin, C. Hao and S. Peiming, "Research on the Method of Features Extraction for Nonstationary Transient Signal Based on EMD Method", International Conference on Communication Software and Networks, Macau, China, (2009) February 27-28, pp. 637- 641.

[11] K. Khaldi and A. O. Boudraa, "On signals compression by EMD", ELECTRONICS LETTERS, vol. 48, Issue 21, (2012) October 11, pp. 1329-1331.

[12] Y. Pei, Y. Wu and D. Jia, "Research on PD Signals Denoising Based on EMD Method", PRZEGLĄD ELEKTROTECHNICZNY (Electrical Review), (2012), pp. 137-140, http://pe.org.pl/articles/2012/1b/30.pdf (Last accessed on February15, 2014). 


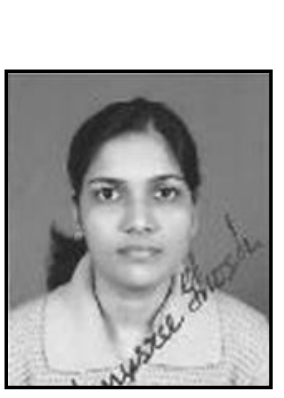

\section{Authors}

Tanusree Ghosh, B.Tech, M.Tech, currently, working as a Ph.D. Research Scholar in the University of Calcutta, Kolkata, India. She was associated as a researcher in West Bengal University of Technology, 2013. She has 2 years experience of Teaching and Research.

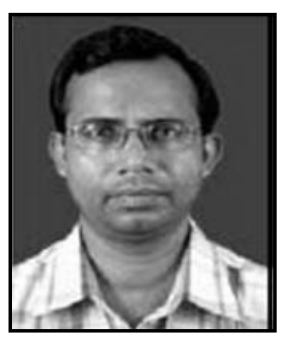

Debnath Bhattacharyya, M.Tech (CSE), Ph.D. (Tech.), currently, associated with Computer Science and Engineering Department, Faculty of Engineering and Technology, NSHM Knowledge Campus - Durgapur, as a Professor and Head. Dr. Bhattacharyya has 17 years of experience in Teaching and Research. He published more than 135 research papers in international Journals and Conferences. He published 4 Text Books for B. Tech, and MCA, so far. He is also associated with West Bengal University of Technology, University of Calcutta and many leading National and International Universities as the Ph.D. Supervisor.

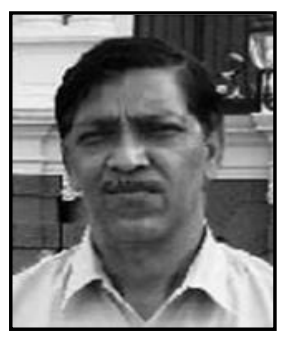

Samir K. Bandyopadhyay, B.E., M.Tech., Ph.D., C.Engg., D.Engg., FIE, FIETE, currently, Professor of Computer Science \& Engineering and Registrar, University of Calcutta, visiting Faculty Dept. of Comp. Sc., Southern Illinois University, USA, MIT, California Institute of Technology, etc. His research interests include Bio-medical Engg, Mobile Computing, Pattern Recognition, Graph Theory, Software Engg.,etc. He has 25 Years of experience at the Postgraduate and undergraduate Teaching \& Research experience in the University of Calcutta. $\mathrm{He}$ has already got several Academic Distinctions in Degree level/Recognition/Awards from various prestigious Institutes and Organizations. He has published 300 Research papers in International \& Indian Journals and 5 leading text books for Computer Science and Engineering. He has visited USA, Finland, Sri Lanka.

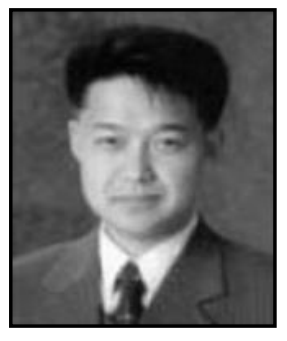

Tai-hoon Kim, M.S., Ph.D. (Electricity, Electronics and Computer Engineering), currently, Professor of Sungshin Women's University, Korea. His research interests include Multimedia security, security for IT Products, systems, development processes, operational environments, etc. He has 18 Years of experience in Teaching \& Research. He has already got distinctive Academic Records in international levels. He has published more than 300 Research papers in International \& National Journals and Conferences. 